\title{
El capital social en la gestión sostenible de la sub- cuenca del río Ulí
}

\author{
The social capital in the sustainable management of the subbasin from Ulí River
}

Jamill Castillo Martínez ${ }^{1}$

\section{Resumen}

La presente investigación se realizó en el Municipio de Siuna, Región Autónoma del Caribe Norte (RACN), en la Subcuenca Ulí, tiene como propósito hacer un análisis sobre la eficiencia del capital social en la gestión integral de la Subcuenca Ulí. Con la participación de 18 comunidades ubicadas alrededor de la subcuenca Ulí: Santa fe, Wany, Santa Rosa, Ulí, Yuluwas, Azadín, Aza, Guzma, El Bambú, Nueva Esperanza, Rosquilete, Copawas, Sikilta, Martha Lorena 1, Martha Lorena, El Guácimo, Saslaysito y Montes de Oro. Este análisis aborda las dimensiones de integración intracomunitaria, conexión extracomunitaria, sinergia institucional y la eficiencia organizativa, en un nivel interpretativo y el método fenomenológico.

En la subcuenca del río Ulí hay un potencial de capital social para la gestión sostenible de la cuenca, con una alta disposición de participación, integración para el desarrollo integral de la misma. Sin embargo, hay una deficiencia en el uso del mismo, puesto que no se generan acciones de confianza, integración y complementación entre las organizaciones comunitarias, así como con las instituciones externas, maximizando la vulnerabilidad de la cuenca y su entorno socio ambiental.

Palabras clave: integración; conexión; sinergia; eficiencia organizativa.

\section{Abstract}

The present investigation was carried out in Siuna Municipality, in the Autonomous Region of the North Caribbean Coast (RACCN), in the Ulí Subbasin, with the purpose of making an analysis on the efficiency of the social capital in the integral management of Ulí Subbasin. With the participation of 18 communities located around the Ulí subbasin: Santa Fe, Wany, Santa Rosa, Ulí, Yuluwas, Azadín, Aza, Guzma, El Bambú, Nueva Esperanza, Rosquilete, Copawas, Sikilta, Martha Lorena 1, Martha Lorena, El Guácimo, Saslaysito and Montes de Oro. This analysis addresses the dimensions of intra community integration, extra community connection, institutional synergy and organizational efficiency, at an interpretive level and the phenomenological method.

In the subbasin of the Uli River there is a potential for social capital for the sustainable management of the basin, with a high willingness to participate, integration for its integral development. However, there is a deficiency in its use, since no trust, integration and complementation actions are generated between community organizations, as well as with external institutions, maximizing the vulnerability of the basin and its socio-environmental context.

Keywords: Integration; connection; synergy; organizational efficiency

\footnotetext{
1 Magíster en Docencia Universitaria, ingeniero Agroforestal, Coordinador del área de Ciencia, Tecnología y Medio Ambiente. Correo electrónico Jasmillc@ yahoo.com; https://orcid.org/0000-0002-3648-9165
}

Recibido: 01/07/2019 - Aprobado: 26/07/2019 


\section{Introducción}

El capital social visto como la telaraña o entramado de relaciones, tanto entre individuos como entre organizaciones, así como la confianza e integración en todos los niveles organizativos, es fundamental para el desarrollo de procesos, como es el de la gestión de cuencas hidrográficas por dos razones esenciales, la primera es que el agua es elemento indispensable para la vida misma por su dependencia para las actividades económicas (doméstico, agropecuaria e industrial) y la segunda, es que quienes habitan en las cuencas son personas, sociedades estructuradas, que hacen uso de los recursos incrementando el deterioro de la misma, pero también pueden y deben ser los responsables de la gestión sostenible de recursos.

Nicaragua, desde el 2010 aprueba una ley de aguas, orientada al manejo integrado de las cuencas, donde establece la creación de un comité de cuencas a nivel nacional, departamental, municipal y comunitario, y mandata a todas las instituciones a trabajar de manera integral con todos los niveles hacia una gestión sostenible de las cuencas, con la finalidad de conservar el agua en calidad y cantidad en el tiempo. Sin embargo, la horizontalidad del proceso todavía está muy débil, pues la participación de las comunidades desde sus propias organizaciones, no es visible, lo que ha llevado un amento del deterioro de las cuencas por el creciente cambio de uso del suelo en las zonas vulnerables y críticas.

Esta investigación hace un análisis profundo desde la visión micro o individual con las dimensiones de integración intracomunitaria, conexión extracomunitaria, y la visión macro u organizacional con las variables de sinergia institucional y eficiencia organizativa, de tal manera que identifica los cuellos de botella en cada variable, y mide de esta manera la existencia y eficiencia del capital social en la gestión de las cuencas hidrográficas, específicamente en la subcuenca del río Ulí.

\section{Revisión de literatura}

Capital social conceptualizado como el contenido de ciertas relaciones y estructuras sociales, es decir, las actitudes de confianza que se dan en combinación con conductas de reciprocidad y cooperación. Ello constituye un capital en el sentido, de que proporciona mayores beneficios a quienes establecen este tipo particular de relaciones y que puede ser acumulado. (Durston, 2002).

Rodríguez (2012). Integra el enfoque del capital social, en calidad de recurso exógeno e individual, inmerso en la red social de un agente, propia de la corriente sociológica de redes, ver figura 1. Con la perspectiva del capital social como un conjunto de relaciones internas, que caracterizan las estructuras de los agentes colectivos (grupos, organizaciones, comunidades, regiones, países, etc.) manteniendo su cohesión y otorgándoles unos beneficios.

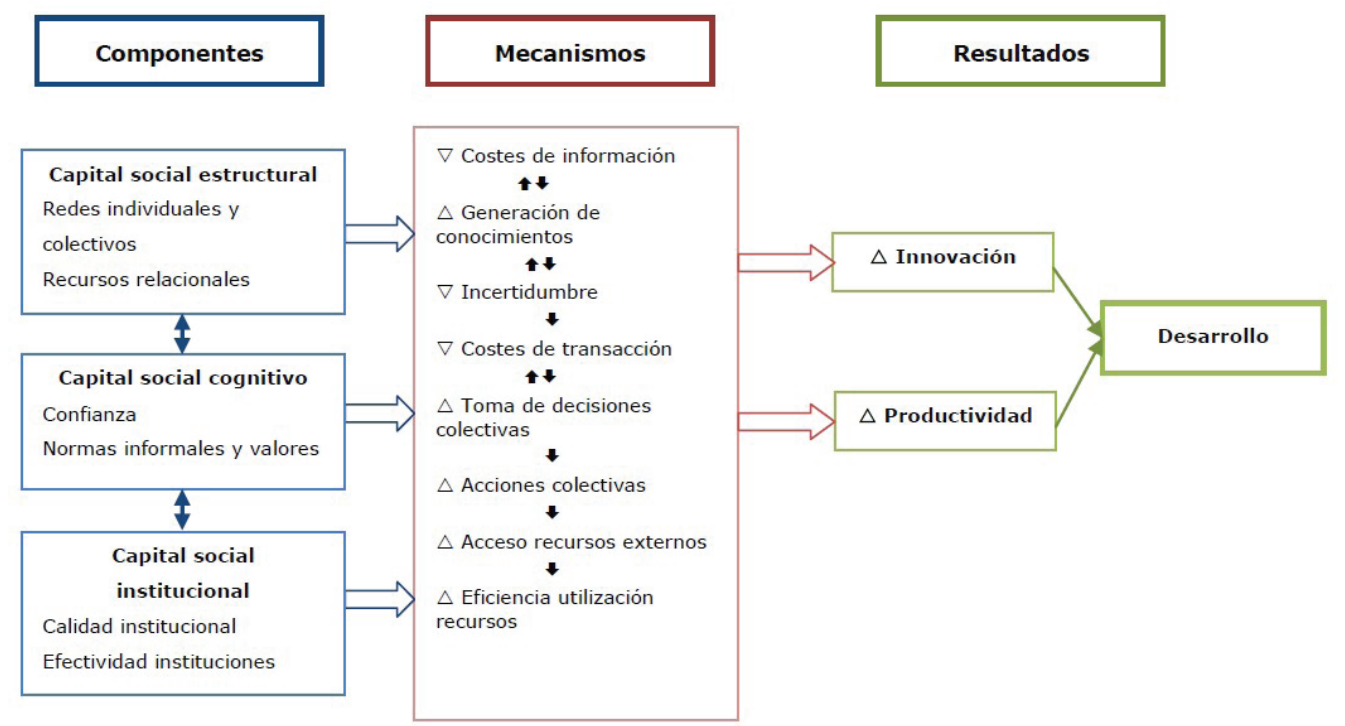

Figura 1. Marco conceptual del capital social y el desarrollo económico. Adaptación Rodríguez (2012). 
Este esquema conceptual es importante, porque en la medida que haya una sinergia entre los componentes del capital social, mayor será el acceso a los recursos y la disponibilidad social, y por lo tanto mejores resultados en los procesos de gestión sostenible de los recursos naturales y el desarrollo del territorio. Esta perspectiva conceptual, es similar a la gestión de los recursos hídricos a nivel de cuencas hidrográficas, puesto que las cuencas tienen que ser vistas y manejadas como un sistema interconectado, tanto en lo ecológico-ambiental como en lo social (ver figura 19), y en esa medida es que se va a lograr una gestión sostenible e integral de las mismas.

En su análisis Woolckoc y Narayan (2000), plantean cuatro visiones para. Para el desarrollo y gestión de los territorios, donde su vinculación e interacción entre comunidades e instituciones son elementos claves para emprender iniciativas de desarrollo. La visión comunitaria, en función de grupos, organizaciones comunitarias en el marco del reconocimiento de los activos sociales de grupos pobre y marginados, la visión de redes, vinculado a empresarios, asociaciones productivas, con el propósito de asegurar un capital social de organizaciones productivas crear puentes de relaciones entre los sectores sociales en gestión del desarrollo con un enfoque de cuencas. La visión institucional tanto pública como privada en función de otorgar libertades, asegurar la transparencia y responsabilidades en los procesos y desarrollo de políticas en la gestión de cuencas hidrográficas. Visión de sinergia, la cual vincula las demás con el propósito de complementar y fomentar la participación, la solidaridad en el marco de relaciones horizontales.

En Guatemala hay una aplicación práctica, sobre la construcción de capital social, desde la perspectiva de organización colectiva/grupal, en la que a partir del convencimiento, primero se crearon organizaciones de grupos, para acceder a recursos que no disponían y generar confianza entre los miembros, posteriormente se logró la asociación de grupos, luego un consejo de coordinación entre comunidades/ aldeas, un comité de coordinación municipal y por ultimo una asociación regional (Durston J., 2002).

El capital social, también ha fomentado procesos de innovación, en el cual al menos hay dos consecuencias positivas, una es considerar la innovación como un proceso social evolutivo del aprendizaje colectivo y la segunda que reduce los costes y falta de coordinación en el desarrollo de procesos y transferencia de tecnologías e investigación.

Rodríguez (2012) refleja que, en la comunidad andaluza, las redes organizacionales son muy escasas, y que están dificultando el tejido productivo. Además, el alcance de las existentes es muy limitado con respecto al territorio, tanto a nivel inter-organizacional como social, dificultando la capacidad de innovación y sus procesos de desarrollo, en este sentido la autora plantea mejorar los siguientes aspectos: Movilizar el capital social, fortalecimiento de los tejidos productivos, la conexión entre empresas, redes empresariales e institucionales y el mejoramiento de la eficacia y eficiencia del marco institucional.

Los grupos de acción local u organizaciones comunitarias en zonas rurales, son de gran importancia para la creación de capital social, agrupando actores claves y fomentando redes entre ellos, esto hace que haya una mayor cohesión y compromiso, generando estrategias comunes compartidas. Sin embargo, estos procesos son a largo plazo, los que se fortalece a medida que los sistemas organizativos van madurando y generan mayor confianza. En este mismo estudio los autores concluyen que es importante el análisis del capital social a partir de redes, tanto en la cohesión interna como la relación a través de puentes externos (Buciega y Esparcia, 2013).

En Nicaragua se puede hablar de la existencia de capital social a partir de 1979 hasta la actualidad, con la creación del cooperativismo para el sector agrario, modelo que se definió en cooperativas agrícolas, cooperativas de crédito y servicio, los cuales tenían una relación de confianza, cooperación y un objetivo común, de la misma manera en la actualidad hay instancias que en teoría por mandato de ley, debería de estar mancomunadas para la gestión de cuencas, como se muestra en la figura 2, para poder ser efectivo en el desarrollo de las comunidades rurales. (Barroso, 2011). 


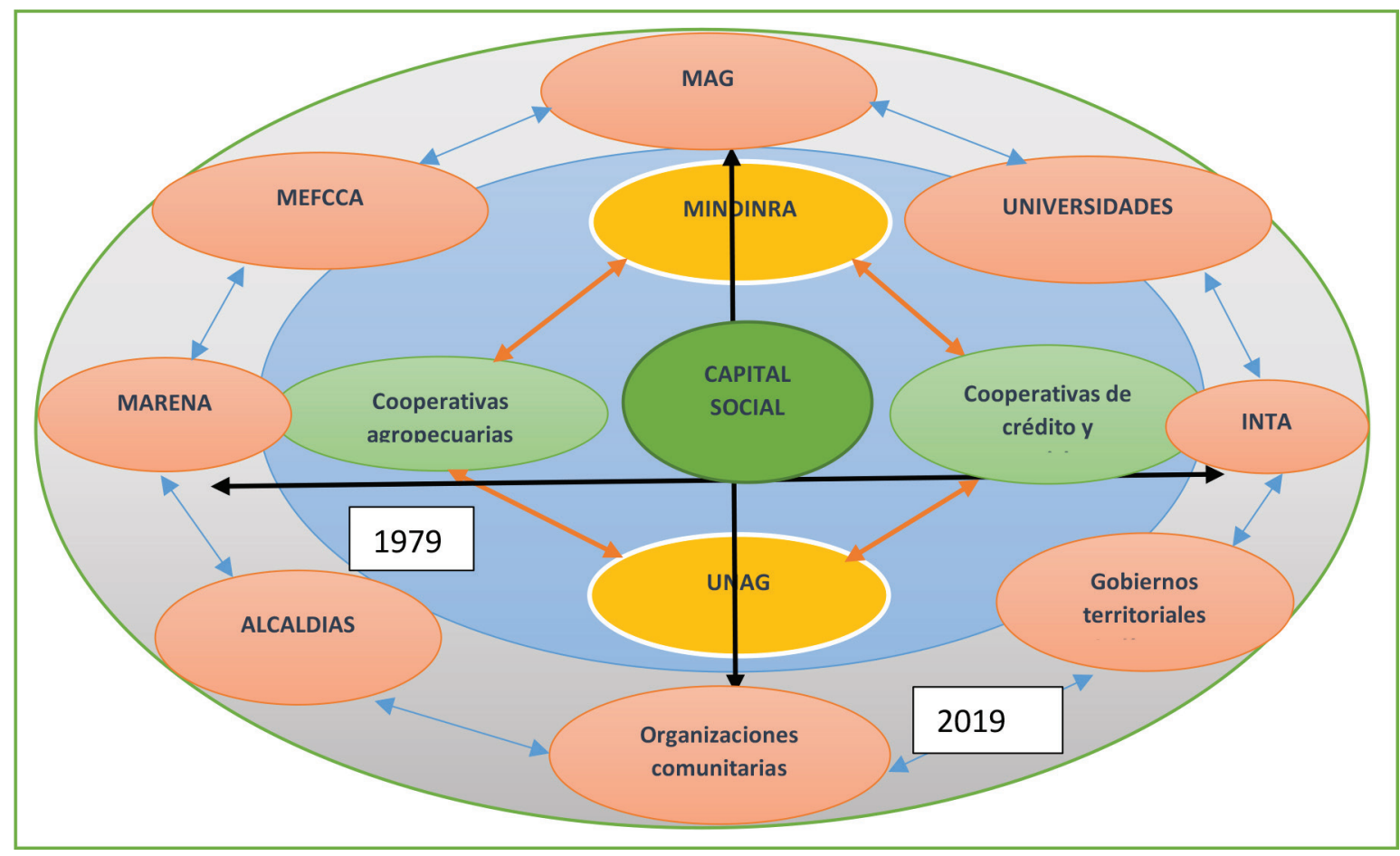

Figura 2. Esquema de las instancias como capital social en Nicaragua para la gestión de cuencas hidrográficas

Desde esta visión, en Nicaragua existe desde el marco legal un entramado de instituciones y organizaciones públicas, privadas y comunitarias, lo que implica que deberíamos tener un capital social consolidado en materia de gestión sostenible de las cuencas hidrográficas y que debería funcionar de manera eficiente. Este aspecto es importante en el sentido de que debía de haber altos niveles de confianza, cooperación de las cooperativas con las demás instancias, para poder acceder a los recursos, esto parte del concepto de capital social que en la actualidad se maneja, como el conjunto de relaciones y confianza que permitan acceder a lo que no se tiene.

En Nicaragua nunca ha habido un modelo de capital social, para la gestión del agua, específicamente con un enfoque de cuencas hidrográficas, Sin embargo, hay un indicio en la zona central norte de Nicaragua en donde por la escasez de agua tanto para consumo humano, beneficios del café y cacao y riego, los agricultores y ciudadanía en conjunto con la municipalidad, están tomando acciones de cooperación para una distribución equitativa y ordenada del uso del agua, así como la gestión para la protección y conservación de la cuenca hidrográfica del macizo de Peñas Blancas. (Gómez, et al, 2011)

Además, en Nicaragua se promueve desde la ley de aguas aprobada en el año 2009, el capital social como elemento integrador en la gestión de los recursos hídricos, el cual establece que debe de haber comités de cuencas en cada comunidad, un consejo de cuencas integrados a partir de los comités comunitarios y comités de cuencas en todos los niveles (municipal, regional y nacional), que deben mantener una relación horizontal, de confianza, responsabilidad, cooperación y participación efectiva en la gestión sostenible de la cuenca.

En la Costa Caribe de Nicaragua, el capital social se presenta como un sistema institucionalizado de cooperación y organización comunitaria, que está vinculado a la correlación familia - comunidad, el cual crea una relación de reciprocidad y confianza entre las personas miembros de la comunidad, entre familias y las relaciones comunales Estas formas de relacionarse de forma tradicional, que no se le llama capital social, es una forma organizada de cooperación, confianza, reciprocidad y pertenencia, que se ajusta claramente al concepto o definiciones de los expertos en capital social (PNUD, 2005). 
Esta manifestación de capital social indígena afro caribeño, comparte el paradigma de la trilogía identificada por Jack (2004) y citada por PNUD (2005) “Juntos tienen, juntos hacen, juntos comparten". Este paradigma "juntos" vincula a cada individuo y comunidad, con el patrimonio de los recursos naturales, y es ahí la importancia del uso y conservación de los mismos, lo que implica, una gestión sostenible milenaria, el cual ha sido elemento fundamental para su sobrevivencia, producto de sus formas de organización ancestral tradicional, la cual se ve amenazada en la actualidad por instituciones y organizaciones externas. (PNUD, 2005)

En la actualidad todos los pueblos indígenas y afrocaribeños han evolucionado, en la parte organizativa y asociatividad, en el que establecen comunidad, territorio y nación como se muestra en la figura 3.

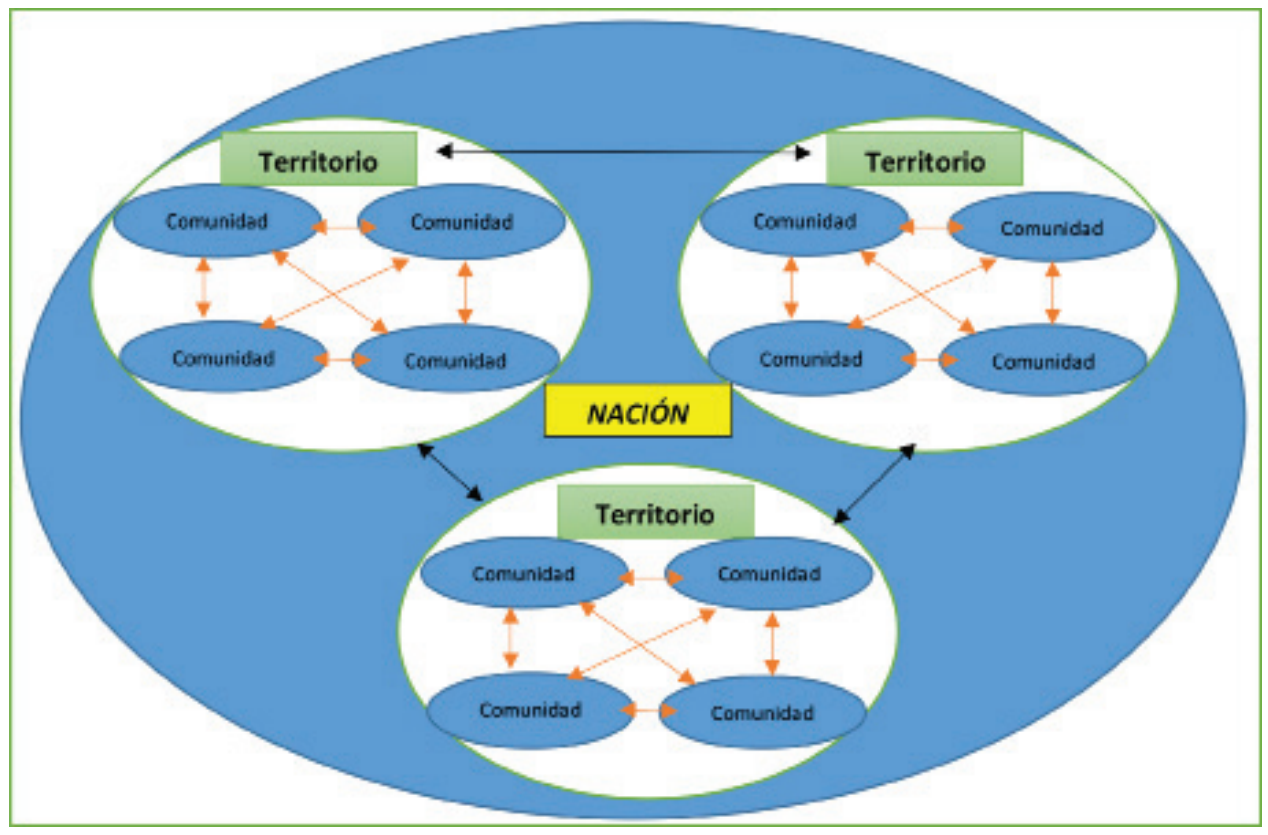

Figura 3. Esquema del capital social indígena mayangna en la Costa Caribe de Nicaragua.

\section{Materiales y métodos}

Esta investigación se realizó en la subcuenca del Ulí, una de las principales que forman la cuenca del Prinzapolka, en la Región Autónoma del Caribe Norte de Nicaragua. Con una extensión de $738.59 \mathrm{Km}^{2}$, delimitada por cuatro municipios (Jinotega, Bocay, Bonanza y Siuna) y tres territorios indígenas (Sauni As, Sauni Bu y Sauni Bas). Esta cuenca tiene el 61\% del territorio en la zona núcleo de la reserva de Biosfera BOSAWAS.

El enfoque sociocrítico con un nivel interpretativo, puesto que hace un análisis de la percepción de las personas sobre las variables de integración, conexión, sinergia y eficiencia organizativa dentro y fuera de la cuenca, con un abordaje desde la perspectiva teórica de la fenomenología, considerando los elementos metodológicos de la interpretación a partir de las relaciones con el contexto tanto interno y externo de los comités de cuencas, así como el método cuantitativo de la encuesta para hacer un mejor abordaje, para lo cual se consideró una muestra de 445 personas para el análisis de la dimensión individual, se realizará una muestra de 445 personas vinculadas las organizaciones locales y municipales, la cual se calculó a través de la fórmula del tamaño de la muestra de poblaciones conocidas $n=\frac{Z^{2}(p * q)}{e^{2}+\frac{\left(Z^{2}(p * q)\right)}{N}}$ y entrevistas al $100 \%$ de las directivas de cada comunidad para la dimensión colectiva organizacional. 


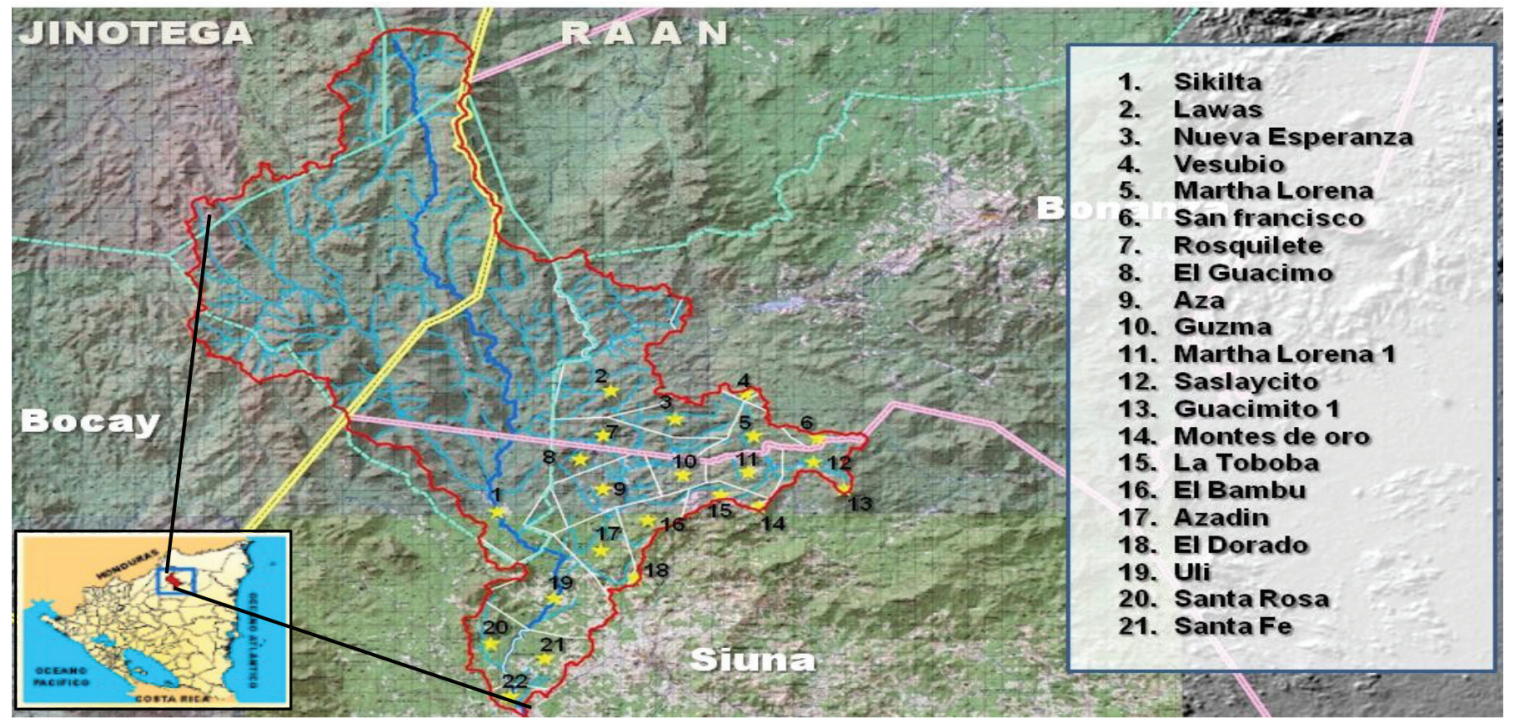

El procesamiento de la información se realizó con el software SPSS y Excel, donde se analizaron las variables de; Integración Intracomunitaria, Conexión extracomunitaria, Sinergia institucional Eficiencia organizativa. Además, se determinó la eficiencia del capital social en la gestión de las cuencas a través de un modelo matemático considerando cada dimensión individual y después en su conjunto, la cual se realizará en cada comunidad y a nivel de la cuenca como territorio. $\mathrm{ECSc}=\mathrm{Iic}+\mathrm{Cec}+\mathrm{Si}+\mathrm{Eo} . \mathrm{A}$ una escala de medición será de o a 1, donde; entre más se acerca al 1, más eficiente es el capital social en la gestión sostenible de cuencas en esa comunidad y el territorio.

\section{Resultados y discusión}

La gestión del agua en Nicaragua está amparada en su marco legal desde la ley 620, ley general de aguas y su reglamento, así como el marco legal para el caso de la Costa Caribe amparada en la ley 28 y ley 445 , que faculta a las regiones autónomas del caribe la autonomía para su administración uso y disfrute de sus recursos naturales, además de la administración del agua a los comités de agua y saneamiento (CAPs), que lo establece la misma ley, desde esta perspectiva la gestión del recurso agua hay una marcada diferencia en la gestión del agua entre el pacifico y el caribe del país. Ver figura 4.

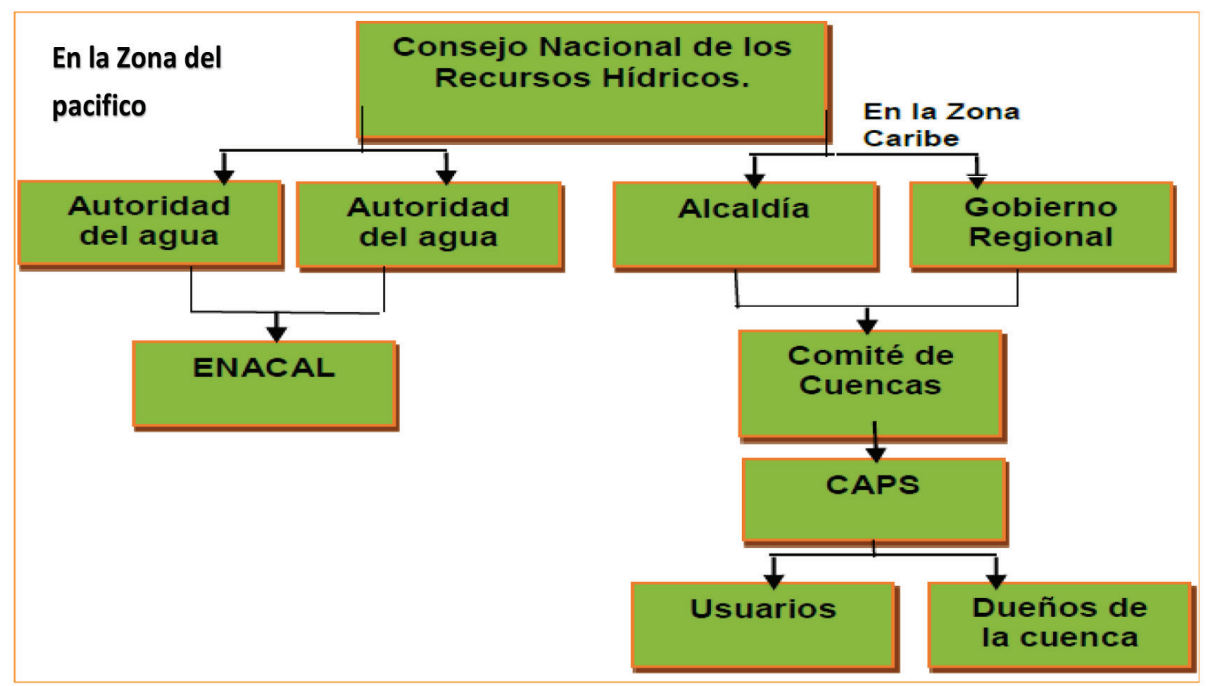

Figura 4. Gestión del agua en Nicaragua 
Sin embargo con una estructura administrativa definida para la gestión con un enfoque de cuenca, esta no ha sido efectiva, Las políticas públicas en la gestión de cuencas, no son integradoras tanto en procesos como en actores, por lo que desde esta perspectiva, conviene enfatizar algo sumamente relevante: las políticas, en sí mismas, no resuelven los problemas, sino que éstos son resueltos por los actores sociales mediante la implementación de sus estrategias, la gestión de sus conflictos y, sobre todo, mediante procesos de aprendizaje permanente. Además de una participación efectiva en ligada a las políticas en materia de gestión de cuencas y a la dinámica de los actores (Aguilar, 2007).

Esta participación efectiva en las políticas públicas de todas las instancias organizativas, en el caso de Nicaragua según la ley, los comités de cuencas comunales, municipales, los organismos de cuencas, la autoridad del agua y el consejo nacional de los recursos hídricos, es fundamental para definir estrategias y acciones consensuadas y comprometidas, es lo que llevaría a avanzar hacia la gestión integrada de los recursos hídricos.

Sin embargo, es ahí donde radica el problema fundamental, que las estructuras no están y si existen no hay coordinación, los comités de agua potable y saneamiento solo existe donde hay un sistema de agua con infraestructura financiada, y solo se vincula al mantenimiento y al servicio del agua como un bien económico, prueba de ello es que en estos comités no hay integrado ningún actor propietario de las zonas donde se recarga el punto de captación, del cual depende la permanencia del sistema.

De manera general la problemática en las cuencas asociadas a lo político institucional está dado por; insuficientes políticas, programas y proyectos con poca participación en materia de uso, conservación y manejo integral del recurso hídrica; reducida participación comunitaria urbana y rural en procesos de gestión socio ambiental; escasa capacidad para generar información básica orientada al manejo del recurso, inadecuada e insuficiente gestión de riesgos; problemas de coordinación entre las diversas iniciativas que se implementan; duplicidad de esfuerzos.

\section{Situación del capital social en la subcuenca del río Ulí}

La situación de la subcuenca referido al capital social existente y su efectividad se a bordo de cuadro dimensiones, la primera sobre la integración intracomunitaria, segunda la conexión extracomunitaria, tercera la sinergia institucional y la cuarta sobre la eficiencia organizativa.

La integración intracomunitaria en la subcuenca del río Ulí, en el que se valoraron los indicadores de participación en la creación de los Comités de Cuencas (CC), Integración de los CC y el nivel de confianza en esos comités, dimensión que alcanzo una puntuación 0.5 , ver figura 5. Lo que implica una deficiente participación de la comunidad.

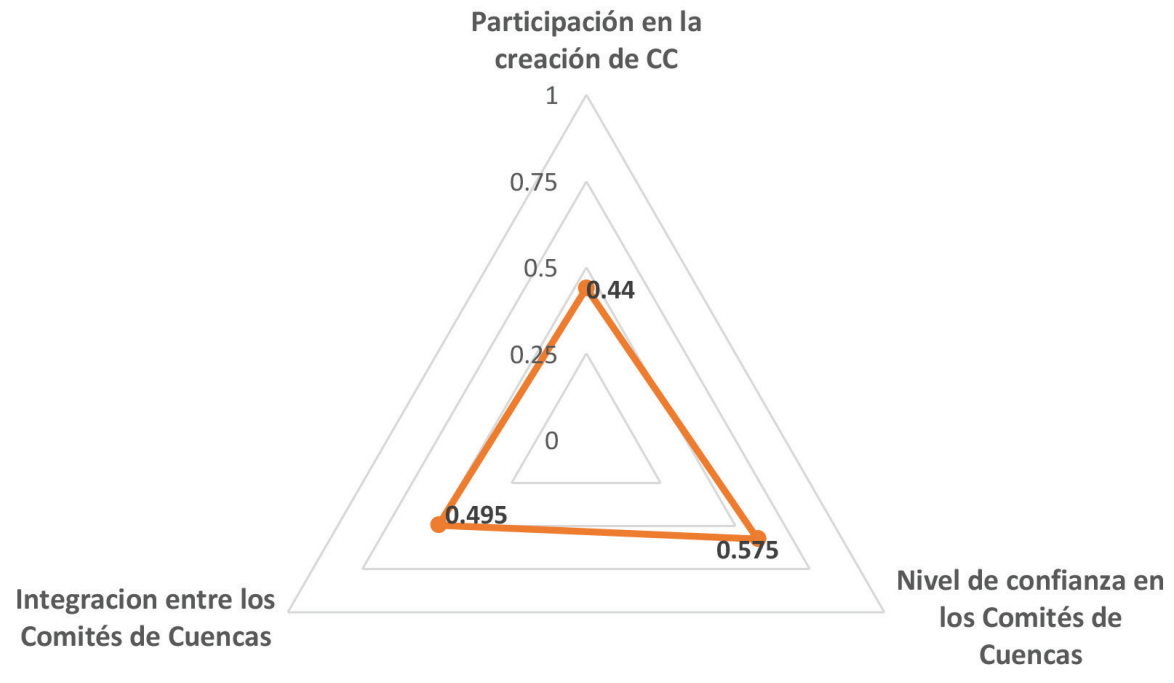

Figura 5. Integración intracomunitaria en la subcuenca del Ulí 
Esta situación desde la perspectiva de los comunitarios, inicia desde la limitada o nula estructura en materia de gestión de cuencas en las comunidades, lo que se visualiza en un nivel de confianza bajo por la paca participación en aspectos relacionados a la gestión de cuencas, por otro lado plantean también que cuando existe al menos un comité de agua potable y saneamiento lo conforman grupos afines o familiares, por lo que la confianza en sus mismas estructuras comunitarias dada vez es más limitada, esta percepción comunitaria de una realidad en estos contextos, cada día debilita un capital social que debería ser muy fuerte, por lo que las personas minimizan sus accionar en materia de gestión, limitándose a su vida cotidiana o a generar nuevos grupos y por consiguiente conflictos comunitarios.

La Conexión extracomunitaria incorpora los indicadores de participación de las instancias comunitarias que trabajan el tema de cuencas o hídrico en las organizaciones externa, las relaciones internas y externas de los comités comunitarios y la confianza que tiene estos en las instituciones externas, en la que se obtuvo una valoración de o.6, ver figura 6 lo que se considera regular.

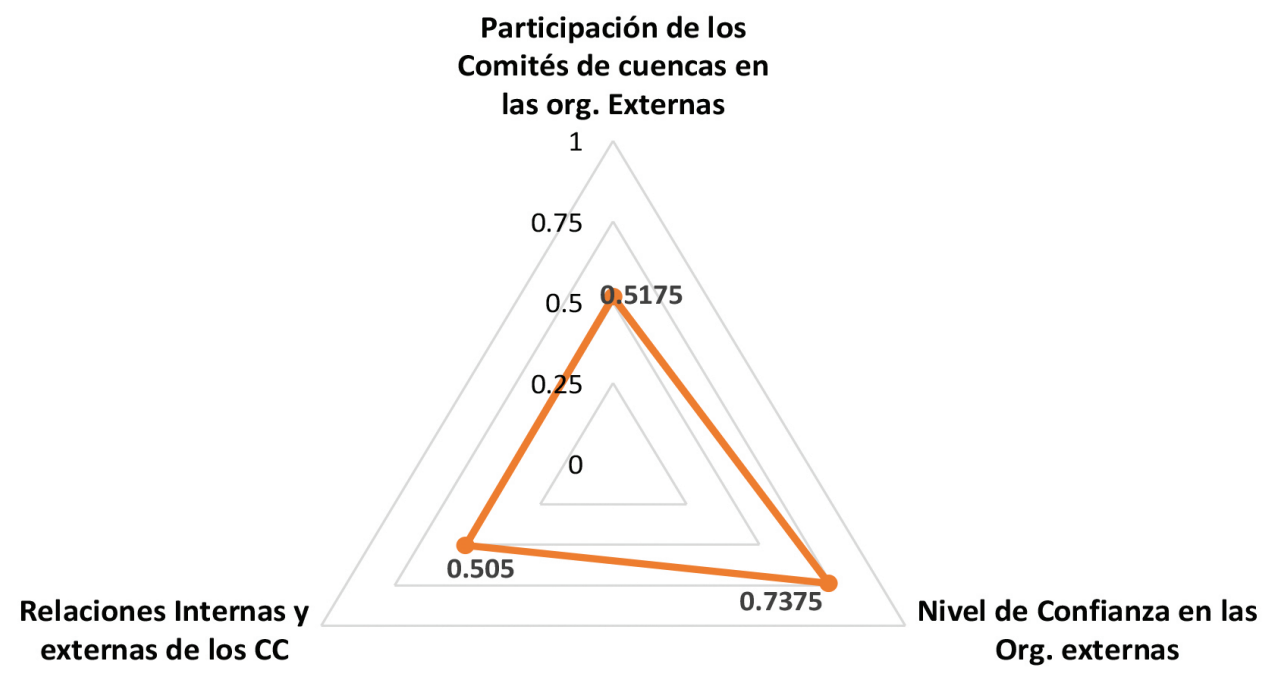

Figura 6. Conexion extracomunitaria en la subcuenca del Ulí en el caribe de Nicaragua

Las conexiones y las vinculaciones que tienen las comunidades con las instituciones que trabajan el tema de manejo de los recursos naturales y el enfoque de cuencas, desde la percepción de las personas todavía hay un buen nivel de confianza en estas para gestionar su manejo, sin embargo, hay muy poca participación comunitaria en estas instancias externas, así como limitada relaciones entre organizaciones comunitarias, esto aumenta la vulnerabilidad y limita el potencial del capital social, puesto que no hay cohesión entre las comunidades y se pierde la confianza sistemáticamente en las instituciones externas y en el liderazgo comunitario, minimizando la gestión efectiva y aumentando los riesgos a las cuencas hidrográficas.

La sinergia institucional aborda un análisis de cooperación, participación e interacción entre las organizaciones comunitarias y con las organizaciones externas para trabajar el enfoque de cuencas en sus procesos de gestión, en la subcuenca del Ulí esta dimensión del capital social, desee la percepción de las personas tiene una valoración de 0.47 ver figura 7.

Esta valoración, indica la limitada sinergia que existe tanto dentro del territorio de una cuenca como con las instituciones que son parte de la gestión de la misma, y esto implica elementos importantes, primero que las instituciones mismas conocen muy poco con abordar la gestión sostenible de cuencas, segundo que hay una limitada organización según como la mandata la ley de aguas lo que minimiza la integración entre las comunidades, tercero, por la falta de organización no hay planificación dentro y fuera de la subcuenca, maximizando la vulnerabilidad lo que acelera el deterioro de la misma, además que 
cuando se logra hacer un proceso de planificación no son partícipes las organizaciones comunitarias, producto de un cuarto elemento que es la nula interacción entre las organizaciones comunitarias y externas en esta temática.

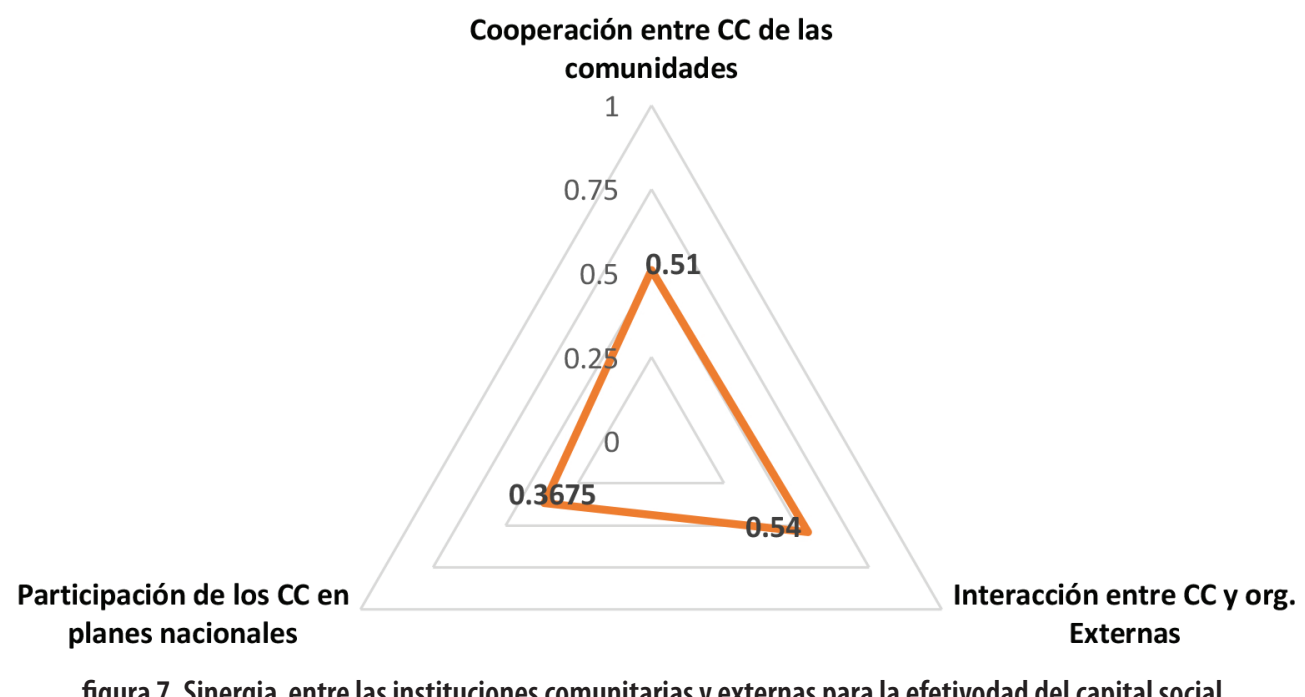

figura 7. Sinergia entre las instituciones comunitarias y externas para la efetivodad del capital social

Esta situación todavía no se ha resuelto, lo que trae consigo un letargo en el capital social existente al estar inactivo, lo que genera un deficiente proceso de gestión de las cuencas hidrográficas, lo que incrementa su deterioro de manera sistemática.

La eficiencia organizativa, vinculada a la valoración, reconocimiento entre las organizaciones comunitarias y externas en la gestión, en la subcuenca del Ulí esta dimensión del capital social tiene una valoración de 0.5, ver figura 8. La cual todavía es deficiente.

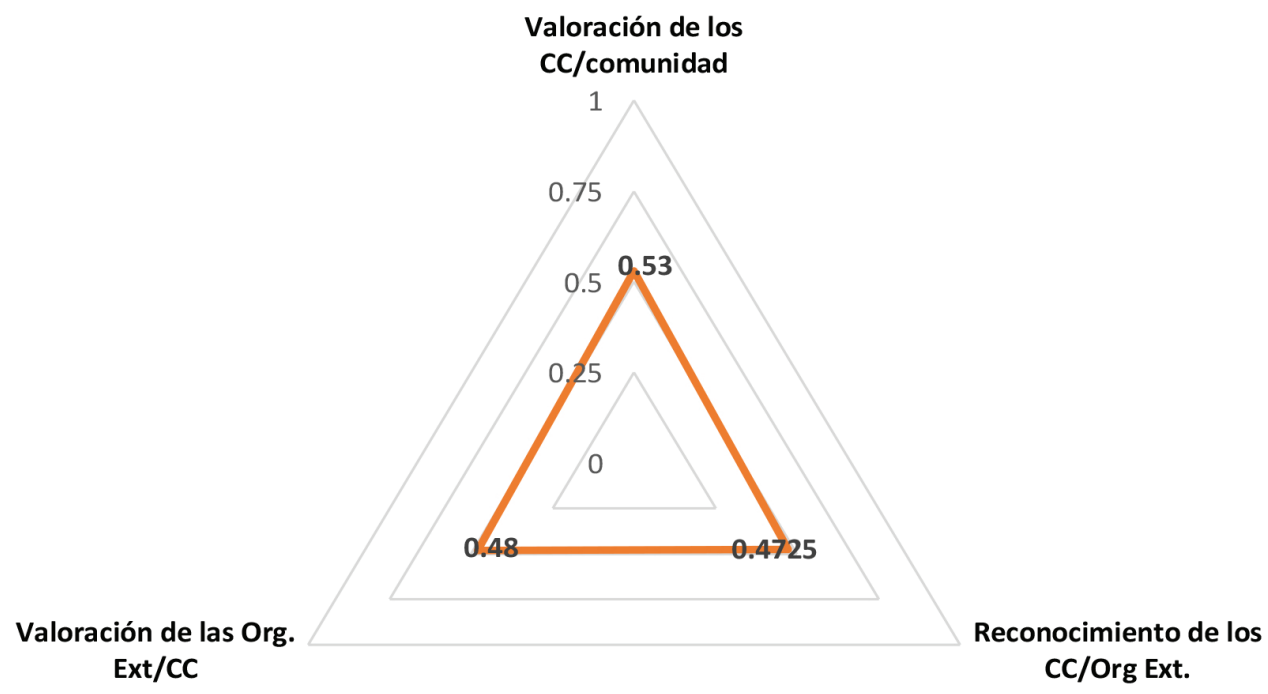

Figura 8. Eficiencia organizativa en la gestión de la subcuenca del Ulí

Esta dimensión del capital social es muy importante, porque es la que genera la confianza que los miembros de una comunidad van a tener en su organización, la que van a tener las organizaciones con las instituciones de estado y no gubernamentales; sin embargo, desde la percepción de las personas que 
habitan la cuenca. Las organizaciones externas no están cohesionadas con la comunidad que solo llegan a decir que hacer y no reconocen no valoran al papel funcional de las organizaciones comunitarias y menos en temas con un enfoque de cuencas.

Esta situación es peligrosa para consolidar un capital social, porque si las comunidades que son parte de una cuenca, sienten que son usadas y que nos parte igual en el proceso de gestión por muchos planes que se realicen no va a tener éxito, esto se debe a que todavía las instituciones mantienen un concepto de saberlo todo y que llegan a enseñar y no a hacer un proceso de gestión conjunto, lo que va a llevar un mal uso de los recursos, al distanciamiento entre comunidad e institución y a lesionar la capital social, lo que provocara el deterioro permanente de la subcuenca.

La eficiencia del capital de manera general en el rango o a 1 es de 0.5 , donde la conexión extracomunitaria es la que llega a ser aceptable con o.6, ver figura 9. En la que todavía los comunitarios y sus organizaciones tienen credibilidad en las organizaciones externas, pero que se está perdiendo paulatinamente, producto de la limitada inserción en los procesos de gestión e integración conjunta.

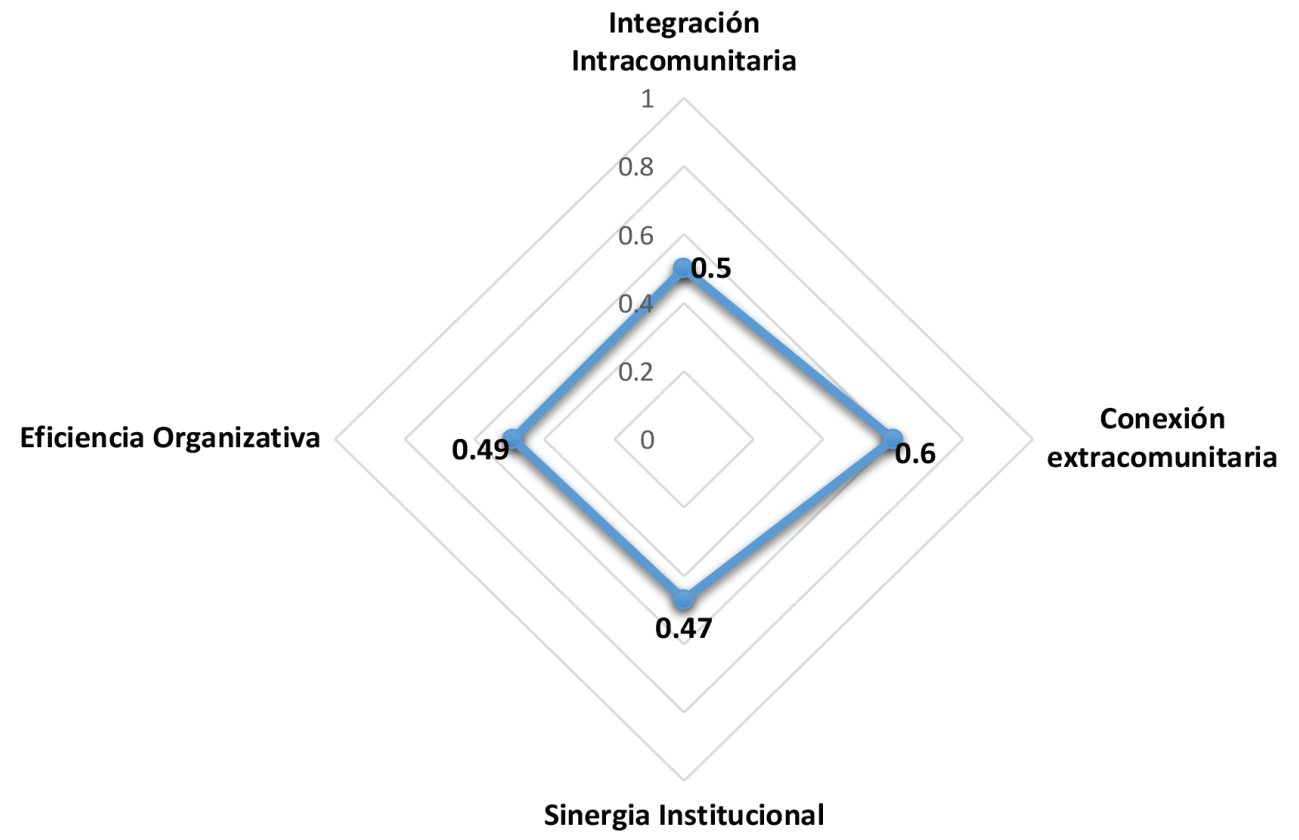

Figura 9. Eficienca del capital social en la subcuenca del Ulí

Esta situación es un punto de considerar, porque se evidencia la pérdida sistemática de la confianza, la credibilidad y la conexión entre las personas y organizaciones que vive en la cuenca y las organizaciones externas, lo que limita la organización, la planificación, la interacción debilitando el capital social y así mismo la gestión sostenible del territorio con un enfoque de cuencas. Esta situación ocurre en Nicaragua, Guatemala y México entre otros países, en la que no se ha podido encontrar un punto de equilibrio para hacer caminar estos procesos.

Desde esta perspectiva hay que considerar que históricamente las comunidades en una cuenca, siempre han estado o tenido una estructura organizativa, participado en sus procesos productivos internos, $y$ han sido las instituciones externas a la cuenca o a las comunidades, en el marco de un instrumento legal de estado que han polarizado a las organizaciones comunitarias por mucho tiempo, ya sea por dimensiones políticas, religiosas, étnicas e incluso de cultura productiva, que hoy en día generar la confianza, la participación efectiva desinteresada es un proceso de mediano o largo plazo y complicado. 
Desde mi percepción, y basado en los principales conceptos en que se fundamentara este análisis, en los planteamientos de las personas que viven en la cuenca, una relación y confianza, la creación de un capital social eficiente debe tener su base principal en la cohesión de las organizaciones comunitarias y posteriormente con las organizaciones externas con un sistema de entramado de telaraña en una cuenca hidrográfica, figura 20.

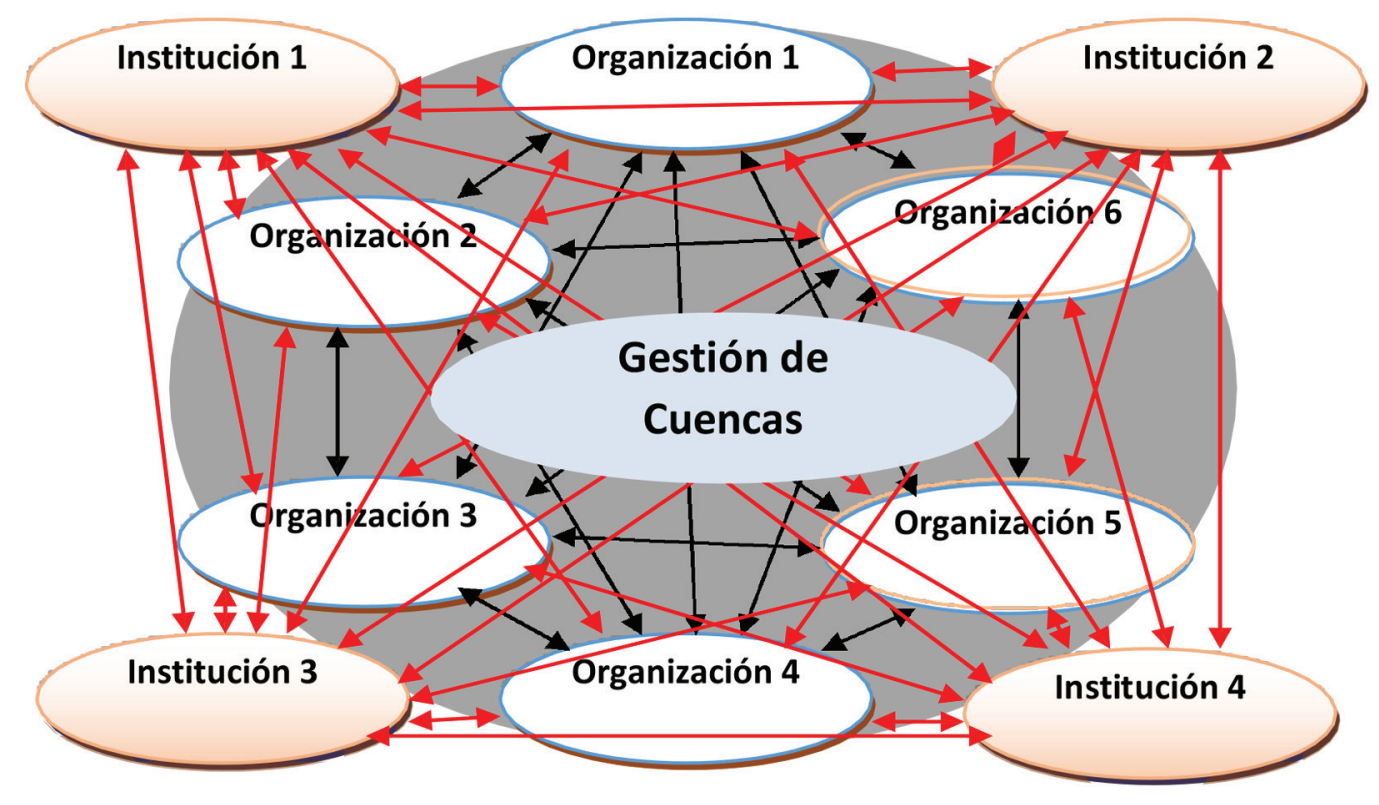

Figura 10. Sinergia, conexión, integración y organización para un capital social efectivo en la gestión de cuencas.

\section{Conclusiones}

El proceso de gestión de cuencas en Nicaragua es diferenciando entre el pacifico y el caribe del país, con la incorporación de la autonomía en la administración y uso del recurso hídrico, a través de gobiernos territoriales indígenas y comunitarios.

El capital social en la subcuenca del río Ulí es deficiente con una valoración de o.5, lo que implica limitaciones en el proceso de integración intracomunitaria, conexión extracomunitaria, sinergia institucional y débil eficiencia organizativa, lo que implica una decadencia del capital social y alta vulnerabilidad a las cuencas hidrográficas. Producto de la poca importancia que las instituciones le dan a esta temática y al incumplimiento administrativo de la ley.

Desde la percepción de las personas y en análisis conceptual para el fortalecimiento el capital social en las cuencas debe tener una base de confianza, la cual debe generarse por el reconocimiento y la integración y organización de las comunidades de la cuenca y las instituciones externas.

\section{Agradecimiento}

Esta publicación obtuvo el financiamiento del Proyecto Ecosistemas Costeros, auspiciado por: Unión Europea, Dka Austria, Cooperación Austriaca para el Desarrollo y Horizontzooo.

\section{Lista de referencias}

Durston , J. (2006). Capital social y gestión participativa en la cuenca de Pátzcuaro. Revista de la CEPAL 90, 106. 
Asamblea Nacional. (2010). Ley General de las Aguas Nacionales: Ley No 620. Managua- Nicaragua.

Barrera, K. (2012). Fortalecimiento del capital social y las capacidades locales para la gestión integral del riesgo en la cuenca Estero San Diego, La Libertad, El Salvador. La Libertad, El Salvador.

Castellón, N. (2009). El comité ejecutivo de la subcuenca del río Jucuapa, Matagalpa, Nicaragua: avances, alcances y aprendizajes. ${ }_{\text {iHacer }}$ buenas cosas y hacerlas bien hechas! Turrialba, C.R.

Castillo, J. (2010). Oferta Hídrica de la cuenca Uly y Madriguera asociado al uso del suelo. URACCAN, Siuna. RAAN.

Cruz, C. (2009). Caracterización biofisica de la Subcuenca Uly,cuenca hidrografica $N^{o} 53$, Jinotega RAAN, Junio 2009. Jinotega, Niacaragua.

Durston, J. (2006). Capital social y gestión participativa en la cuenca de Pátzcuaro. Revista de la CEPAL 90, 106.

Faustino, J. (2013). Innovacion, aprendizaje y comunicacion para la cogestion adaptativa de cuencas.

Flores, O. (2012). Diagnostico socio economico y ambiental, parte media- baja de la Sub cuenca Uly,Siuna 2012. Siuna, RACN.

Garcia, D. (2010). Análisis de la gobernanza del recurso hídrico en la subcuenca del río Ulí,reserva de la Biosfera Bosawas, Nicaragua. Turrialba, Costa Rica.

García, Y. (2012). Analisis del capital social en la gestion del recurso hidrico y su influencia en la cohesion social de la comunidad de Perdernal de Puriscal 2011. Heredia, Costa Rica.

López, E. (2013). Capital social para la gestion integral del agua en la Cuenca Chili, Peru. Jiutepec Morelos, Mexico.

Márquez, D. (2009). El capital social en la Vega Media Sevilla: un nuevo elemento del desarrollo. España.

Morales, J. (1998). Manejo de cuenca. Managua, Nicaragua.

Moyano, E. (2003). Capital social, gobernanza y desarrollo rural. Cordoba, España.

Moyano., E. (2006). El asocianismo en el sector agroalimentario y su contribución a la generación del capital social. Córdoba España: IESA.

Pérez , R. (2006). Análisis del proceso hacia la cogestión en la subcuenca del río Aguas Calientes, departamento de Madriz, Nicaragua. Madriz, Nicaragua.

Programa Focuenca 11. (2009). Modelo de cogestión adaptativa de cuencas hidrograficas Subcuenca aguas caliente. Madriz, Nicaragua.

Reyes, B. (2008). Creación y funcionamiento del Comité de Cuenca Bimunicipal Aguas Calientes: Sistematización de experiencias. Turrialba, C.R.

Troval, A. (2009). Misión de Apoyo para la Elaboración de una propuesta de estratégica de acciones a corto y mediano plazo para el manejo integrado de la subcuenca del Río Uli, Siuna, RAAN. Siuna, RACN.

Umaña, E. (2002). Taller de capacitacion: Educacion ambiental con enfoque en manejo de cuencas y prevencion de desastres. San Nicolas, Esteli. 